\title{
Pacific
}

Journal of

Mathematics

\section{BRANCHED COVERINGS OF SURFACES WITH AMPLE COTANGENT BUNDLE}

\author{
MichaEL JEROME SPURR
}




\title{
BRANCHED COVERINGS OF SURFACES WITH AMPLE COTANGENT BUNDLE
}

\author{
Michael J. SpurR
}

\begin{abstract}
Let $f: X \rightarrow Y$ be a branched covering of compact complex surfaces, where the ramification set in $X$ consists of smooth curves meeting with at most normal crossings and $Y$ has ample cotangent bundle. We further assume that $f$ is locally of form $(u, v) \rightarrow\left(u^{n}, v^{m}\right)$. We characterize ampleness of $T^{*} X$. A class of examples of such $X$, which are branched covers of degree two, is provided.
\end{abstract}

1. Introduction. An interesting problem in surface theory is the construction and characterization of surfaces with ample cotangent bundle. They are necessarily algebraic surfaces of general type. Natural examples occur among the complete intersection surfaces of abelian varieties. More subtle examples are those constructed by Hirzebruch [6] using line-arrangements in the plane. The characterization of those of Hirzebruch's line-arrangement surfaces with ample cotangent bundle is due to Sommese [8]. In this article, we will give a characterization of ampleness of the cotangent bundle of a class of surfaces which branch cover another surface with ample cotangent bundle. We will also construct certain branched coverings of explicit line-arrangement surfaces; these constructions will again have ample cotangent bundle.

For any vector bundle $E$ over a base manifold $M$, the projectivization $\mathbf{P}(E)$ is a fiber bundle over $M$, with fiber $\mathbf{P}_{q}(E)$ over $q \in M$ given by $\mathbf{P}_{q}(E) \approx\left(E_{q}^{*} \backslash 0\right) / \mathbf{C}^{*}$. There is a tautological linebundle $\xi_{E}$ over $\mathbf{P}(E)$ satisfying (i) $\left.\xi_{E}\right|_{\mathbf{P}_{q}(E)} \approx O(1)_{\mathbf{P}_{q}(E)} \forall q \in M$, and (ii) the projection $\rho_{E}: \mathbf{P}(E) \rightarrow M$ gives $\rho_{E_{*}}\left(\xi_{E}\right) \approx E$. In the case that $E=T^{*} X$ we will denote $\rho_{E}=\rho_{T^{*} X}$ simply by $\rho$.

Definition. The vector bundle $E$ is ample if $\xi_{E}$ over $\mathbf{P}(E)$ is ample.

In $\S 2$ we prove preliminary results along with:

THEOREM 1.1. Let $X$ and $Y$ be compact complex surfaces, with $Y$ having ample cotangent bundle. Let $f: X \rightarrow Y$ be a branched covering which can be locally represented with coordinate charts of form $f:(u, v) \rightarrow\left(u^{n}, v^{m}\right)$. Let $f$ have ramification set $\bigcup B_{j}$ in $X$ 
consisting of smooth curves meeting in normal crossings. Let $\cup C_{\alpha}$ be the branch locus in $Y$. Then:

$$
T^{*} X \text { is ample } \Leftrightarrow B_{j} \cdot B_{j}<0 \forall j \Leftarrow C_{\alpha} \cdot C_{\alpha}<0 \forall \alpha .
$$

In $\S 3$ we give explicit examples of Theorem 1.1 which lie in a class of degree 2 branched covers.

In what follows $e(C)$ will denote the euler number of a curve $C$.

2. Ample cotangent bundles. Let $f: X \rightarrow Y$ be a branched covering of compact complex surfaces, with $T^{*} Y$ ample and with ramification set $\bigcup B_{j}$ in $X$ consisting of smooth curves meeting in normal crossings. Note that $T^{*} Y$ ample gives that the canonical bundle $K_{Y}$ is ample [4]. Hence $Y$ is projective algebraic, which gives that $X$ is also projective algebraic [1], and in turn that $\mathbf{P}\left(T^{*} X\right)$ is also projective algebraic. Let $f$ be locally represented with coordinate charts of form $f:(u, v) \rightarrow\left(u^{n}, v^{m}\right)$. The differential $f_{*}: T X \rightarrow T Y$ induces a meromorphic mapping $F: \mathbf{P}\left(T^{*} X\right) \rightarrow \mathbf{P}\left(T^{*} Y\right)$ given by $F(x,[w])=\left(f(x),\left[f_{*}(w)\right]\right)$, where $[w]$ denotes the line in the tangent bundle containing the tangent vector $w$. The indeterminacy set $I$ of $F$ corresponds to [w] such that $f_{*}(w)=0$. Blowing up $I$ to resolve the indeterminacy of $f$ (see [5], [10]) one gets $\Pi\left(T^{*} X\right)$ and obtains $b: \Pi\left(T^{*} X\right) \rightarrow \mathbf{P}\left(T^{*} X\right)$ and $\Phi: \Pi\left(T^{*} X\right) \rightarrow \mathbf{P}\left(T^{*} Y\right)$, holomorphic, with $F \circ b=\Phi$ on $\Pi\left(T^{*} X\right) \backslash b^{-1}(I)$. Let $E=b^{-1}(I)$ be the exceptional set over $I$ in $\Pi\left(T^{*} X\right)$. We need to precisely describe the indeterminacy set $I$. Before proceeding, we mention that, in the case that $f$ is locally of form $(u, v) \rightarrow\left(u^{n}, v^{m}\right)$, over each curve $B_{j}$ in the ramification set in $X$ there is a splitting of $T X$, due to Sommese [8], [9], namely $\left.T X\right|_{B_{j}} \approx T B_{j} \oplus N_{B_{j}}$. In particular $N_{B_{j}}$, the normal bundle to $B_{j}$, is a subbundle of $\left.T X\right|_{B_{j}}$, and the pair $B_{j}, N_{B_{j}}$ gives a curve $\widetilde{B}_{j}$ in $\mathbf{P}\left(T^{*} X\right)$.

Proposition 2.1. The indeterminacy set I of $F: \mathbf{P}\left(T^{*} X\right) \rightarrow \mathbf{P}\left(T^{*} Y\right)$ is of form

$$
I=\bigcup_{j} \widetilde{B}_{j} \cup \bigcup_{\alpha} F_{\alpha}
$$

where the $F_{\alpha}$ are fibers of $\rho$ and the $\widetilde{B}_{j}$ are the curves in $\mathbf{P}\left(T^{*} X\right)$ corresponding to the pairs $B_{j}$ and $N_{B_{j}}$ for $B_{j}$ in the ramification set of $X$.

Proof. In local coordinates let $f: X \rightarrow Y$ be given by $f(u, v)=$ $\left(u^{n}, v^{m}\right)$. Then note that the indeterminacy set in $\mathbf{P}\left(T^{*} X\right)$ locally 
corresponds to the (non-zero) annihilators in $\operatorname{ann}\left\langle d\left(u^{n}\right), d\left(v^{m}\right)\right\rangle:=$ $\left\{w \in T X \mid d\left(u^{n}\right)(w)=0\right.$ and $\left.d\left(v^{m}\right)(w)=0\right\}$. By analyzing the Jacobian $f_{*}$ one sees that $\operatorname{ann}\left\langle d\left(u^{n}\right), d\left(v^{m}\right)\right\rangle=\left\{w \in T X \mid f_{*}(w)=0\right\}$. Therefore locally $I=\left\{\operatorname{ann}\left\langle d\left(u^{n}\right), d\left(v^{m}\right)\right\rangle \backslash 0\right\} / \mathbf{C}^{*}$. If both $n, m>1$ then $I$ includes the whole fiber $\rho^{-1}(0,0)$ giving an $F_{\alpha}$. If only one of $n, m$ is greater than 1 , say $n>1$ with $m=1$, then the curve $B_{1}$ corresponding to $u=0$ is in the ramification set and ann $\left\langle d\left(u^{n}\right), d\left(v^{m}\right)\right\rangle$ corresponds to the normal bundle of $B_{1}$ via Sommese's splitting lemma [8], [9]. Thus $B_{1}$ and $N_{B_{1}}$ determine $\widetilde{B}_{1}$. Similarly one obtains the remaining $\widetilde{B}_{j}$.

Let $\xi_{1}$ be the tautological bundle over $\mathbf{P}\left(T^{*} X\right)$ and let $\xi_{2}$ be the tautological bundle over $\mathbf{P}\left(T^{*} Y\right)$, as in the second paragraph of $\S 1$. Then $b^{*}\left(\xi_{1}\right)$ on $\Pi\left(T^{*} X\right)$ relates to $\Phi^{*}\left(\xi_{2}\right)$ in a key manner via the following:

Proposition 2.2. $b^{*}\left(\xi_{1}^{-1}\right)+D=\Phi^{*}\left(\xi_{2}^{-1}\right)$ where $D=\sum n_{\alpha} D_{\alpha}$ is an effective divisor on $\Pi\left(T^{*} X\right)$ supported on the exceptional set $E$ of $\Pi\left(T^{*} X\right)$.

Proof. $f_{*}: T X \rightarrow T Y$ given by $(x, w) \rightarrow\left(f(x), f_{*}(w)\right)$ induces $F: \mathbf{P}\left(T^{*} X\right) \rightarrow \mathbf{P}\left(T^{*} Y\right)$ which is given by $(x,[w]) \rightarrow\left(f(x),\left[f_{*}(w)\right]\right)$. Here $x \in X, w$ is a tangent vector at $x$, and [w] denotes the line in the tangent bundle containing $w . F$ has indeterminacy set $I$ as described in Proposition 2.1. Over $\mathbf{P}\left(T^{*} X\right) \backslash I, f$ induces the mapping $f_{*}: \xi_{1}^{-1} \rightarrow \xi_{2}^{-1}$ given by $(x,[w], w) \rightarrow\left(f(x),\left[f_{*}(w)\right], f_{*}(w)\right)$. This in turn yields the globally defined holomorphic mapping over $\Pi\left(T^{*} X\right) \beta: b^{*}\left(\xi_{1}^{-1}\right) \rightarrow \xi_{2}^{-1}$ given by $(p, w) \rightarrow(f(\rho(b(p))), \Phi(p)$, $\left.f_{*}(w)\right)$ where $p \in \Pi\left(T^{*} X\right), b(p)=[w]$, and $\rho(b(p))=x$. Furthermore $\Phi(p)=\left[f_{*}(w)\right]$ if $f_{*}(w) \neq 0$ (i.e. off $\left.b^{-1}(I)\right)$. In turn, $\beta$ gives the mapping $\gamma$ over $\Pi\left(T^{*} X\right) \gamma: b^{*}\left(\xi_{1}^{-1}\right) \rightarrow \Phi^{*}\left(\xi_{2}^{-1}\right)$ given by $(p, w) \rightarrow\left(p, \Phi(p), f_{*}(w)\right)$. There is vanishing of $f_{*}(w)$ over $E=b^{-1}(I)$, giving $D$.

We will prove Theorem 1.1 using the Nakai Criterion for ampleness [7]: the holomorphic line bundle $\xi_{1}$ on the projective algebraic manifold $P\left(T^{*} X\right)$ is ample if and only if for every subvariety $V_{n}$ of dimension $n \leq \operatorname{dim} \mathbf{P}\left(T^{*} X\right)$ one has that $\int_{V_{n}} c_{1}^{n}\left(\xi_{1}\right)>0$. For brevity we define $\xi_{1}^{n} \cdot V_{n}:=\int_{V_{n}} c_{1}^{n}\left(\xi_{1}\right)$.

Proof of Theorem 1.1. Assume that $C_{\alpha} \cdot C_{\alpha}<0$ for each $C_{\alpha}$ in the branch locus in $Y$. We show that $B_{j} \cdot B_{j}<0$ for all $B_{j}$ in the 
ramification set. Let $\pi^{*}\left(C_{\alpha}\right)=\sum_{k} n_{\alpha k} B_{\alpha k}$. Then

$$
\begin{aligned}
B_{\alpha j} \cdot \pi^{*}\left(C_{\alpha}\right) & =B_{\alpha j} \cdot \sum_{k} n_{\alpha k} B_{\alpha k} \\
& =n_{\alpha j} B_{\alpha j} \cdot B_{\alpha j}+B_{\alpha j} \cdot \sum_{k \neq j} n_{\alpha k} B_{\alpha k}=\operatorname{deg}\left(\left.\pi\right|_{B_{\alpha j}}\right) C_{\alpha} \cdot C_{\alpha}
\end{aligned}
$$

and hence

$$
B_{\alpha j} \cdot B_{\alpha j}=n_{\alpha j}^{-1} \operatorname{deg}\left(\left.\pi\right|_{B_{\alpha j}}\right) C_{\alpha} \cdot C_{\alpha}-n_{\alpha j}^{-1} B_{\alpha j} \cdot \sum_{k \neq j} n_{\alpha k} B_{\alpha k}<0
$$

giving the implication.

Assume next that $T^{*} X$ is ample. We show that $B_{j} \cdot B_{j}<0$ for any $B_{j}$ in the ramification set in $X$. Now for any $B_{j}$ in the ramification set in $X$ the splitting lemma of Sommese [8], [9] gives that $\left.T X\right|_{B_{j}} \approx$ $T B_{j} \oplus N_{B_{j}}$. For $\widetilde{B}_{j}$ the curve in $\mathbf{P}\left(T^{*} X\right)$ determined by $B_{j}$ along with $N_{B_{j}}$, we have $0>\xi_{1}^{-1} \cdot \widetilde{B}_{j}=N_{B_{j}} \cdot B_{j}=B_{j} \cdot B_{j}$.

Conversely, assume that $B_{j} \cdot B_{j}<0$ for each $B_{j}$ in $\bigcup B_{j}$. We show that $T^{*} X$ is ample. First note that since $T^{*} Y$ is ample we have that $e\left(f\left(B_{j}\right)\right)$ is negative; hence by Riemann-Hurwitz $e\left(B_{j}\right) \leq e\left(f\left(B_{j}\right)\right)<$ 0 . To prove ampleness of $T^{*} X$ (i.e. of $\xi_{1}$ ) we show that $\xi_{1}^{n} \cdot V_{n}>0$ for all subvarieties $V_{n}$ in $\mathbf{P}\left(T^{*} X\right)$ where $\xi_{1}$ is the tautological bundle over $\mathbf{P}\left(T^{*} X\right)$. We handle the three cases $n=1,2,3$ separately.

Case (1) $n=1$. Let $V_{n}=C$ be an effective irreducible curve in $\mathbf{P}\left(T^{*} X\right)$. We show that $C \cdot \xi_{1}^{-1}<0$. This is accomplished in three sub-cases:

Case (1i). Suppose that $\rho^{-1}\left(\bigcup B_{j}\right) \not \supset C$. Let $C^{\prime}$ be the proper transform of $C$ in $\Pi\left(T^{*} X\right)$. By Proposition $2.2, \Phi^{*}\left(\xi_{2}^{-1}\right) \cong b^{*}\left(\xi_{1}^{-1}\right)+$ $D$ where $D=\sum_{\alpha} n_{\alpha} D_{\alpha}$ is an effective divisor. So

$$
\begin{aligned}
C \cdot \xi_{1}^{-1} & =C^{\prime} \cdot b^{*}\left(\xi_{1}^{-1}\right)=C^{\prime} \cdot\left(\Phi^{*}\left(\xi_{2}^{-1}\right)-D\right) \\
& =\operatorname{deg}\left(\left.\Phi\right|_{C^{\prime}}\right) \Phi\left(C^{\prime}\right) \cdot \xi_{2}^{-1}-C^{\prime} \cdot D<0 .
\end{aligned}
$$

The last inequality follows since: $\Phi\left(C^{\prime}\right)$ is a curve in $P\left(T^{*} Y\right), \xi_{2}$ is ample (which gives that $\Phi(C) \cdot \xi_{2}^{-1}<0$ ), and $\left.D\right|_{C^{\prime}}$ is effective on $C^{\prime}$ as $C^{\prime}$ is not contained in $D$.

Case (1ii). Suppose that $C$ is a fiber of $\rho$ (and therefore $\left.C \cong \mathbf{P}^{1}\right)$. Then $C \cdot \xi_{1}^{-1}=-1<0$, since $\left.\xi_{1}\right|_{C} \cong O(1)$. 
Case (1iii). Suppose $C$ is contained in $\rho^{-1}\left(\cup B_{j}\right)$ but is not a fiber of $\rho$. Then $\rho(C)=B_{j}$ for some $j$. For $\nu: \eta C \rightarrow C$ the normalization of $C$, one has that $\rho \circ \nu: \eta C \rightarrow \rho(C)$. One has the vector bundle maps:

$$
0 \rightarrow \nu^{*}\left(\left.\xi_{1}^{-1}\right|_{C}\right) \rightarrow \nu^{*} \rho^{*}\left(\left.T X\right|_{\rho(C)}\right)
$$

and

$$
0 \rightarrow \nu^{*} \rho^{*} T \rho(C) \rightarrow \nu^{*} \rho^{*}\left(\left.T X\right|_{\rho(C)}\right) \rightarrow \nu^{*} \rho^{*} N \rho(C) \rightarrow 0 .
$$

Hence one of the sequences of sheaves (2.1) or (2.2) below must be valid.

$$
\begin{aligned}
& 0 \rightarrow \nu^{*} \xi_{1}^{-1} \rightarrow \nu^{*} \rho^{*} T \rho(C) \rightarrow Z_{1} \rightarrow 0, \\
& 0 \rightarrow \nu^{*} \xi_{1}^{-1} \rightarrow \nu^{*} \rho^{*} N \rho(C) \rightarrow Z_{2} \rightarrow 0,
\end{aligned}
$$

where $Z_{1}$ and $Z_{2}$ are sheaves with finite support on $\eta C$. By letting $M_{1}=\nu^{*} \rho^{*} T \rho(C)$ and $M_{2}=\nu^{*} \rho^{*} N \rho(C)$ we rewrite (2.1) and (2.2) as:

$$
0 \rightarrow \nu^{*} \xi_{1}^{-1} \rightarrow M_{i} \rightarrow Z_{i} \rightarrow 0 .
$$

By utilizing the long exact sequence associated to (2.3), along with Riemann-Roch, one concludes that:

$$
\begin{aligned}
c_{1}\left(\xi_{1}^{-1}\right) \cdot C & =c_{1}\left(\nu^{*} \xi_{1}^{-1}\right) \cdot \eta C \\
& \leq c_{1}\left(M_{i}\right) \cdot \eta C=\left\{\begin{array}{l}
\operatorname{deg}(\rho \circ \nu) c_{1}(T \rho(C)) \cdot \rho(C) \\
\operatorname{deg}(\rho \circ \nu) c_{1}(N \rho(C)) \cdot \rho(C) .
\end{array}\right.
\end{aligned}
$$

Now $c_{1}(T \rho(C)) \cdot \rho(C)=e(\rho(C))=e\left(B_{j}\right)<0$. We have that

$$
c_{1}(N(\rho(C))) \cdot \rho(C)=\rho(C) \cdot \rho(C)=B_{j} \cdot B_{j}<0
$$

by hypothesis. Therefore in all cases $\xi_{1}^{-1} \cdot C<0$. So $\xi_{1} \cdot C>0$ and Case 1 is proven.

Case (2) $n=2 . V_{n}=S$ is an effective irreducible surface in $\mathbf{P}\left(T^{*} X\right)$. Let $\Sigma$ be the proper transform of $S$ in $\Pi\left(T^{*} X\right)$.

Case (2i) $\Phi(\Sigma)$ is 2 dimensional.

$$
\begin{aligned}
S \cdot \xi_{1}^{2} & =S \cdot\left(\xi_{1}^{-1}\right)^{2}=\Sigma \cdot\left(b^{*}\left(\xi_{1}^{-1}\right)\right)^{2}=\Sigma \cdot\left(\Phi^{*}\left(\xi_{2}^{-1}\right)-D\right)^{2} \\
& =\Sigma \cdot \Phi^{*}\left(\xi_{2}^{-1}\right) \cdot\left(\Phi^{*}\left(\xi_{2}^{-1}\right)-D\right)-\Sigma \cdot D \cdot\left(\Phi^{*}\left(\xi_{2}^{-1}\right)-D\right) \\
& =\Sigma \cdot \Phi^{*}\left(\xi_{2}^{-1}\right)^{2}-\Sigma \cdot \Phi^{*}\left(\xi_{2}^{-1}\right) \cdot D-\Sigma \cdot D \cdot\left(\Phi^{*}\left(\xi_{2}^{-1}\right)-D\right) .
\end{aligned}
$$


So

$$
\begin{aligned}
S \cdot \xi_{1}^{2}= & \operatorname{Deg}\left(\left.\Phi\right|_{\Sigma}\right) \Phi(\Sigma) \cdot\left(\xi_{2}^{-1}\right)^{2} \\
& -\Sigma \cdot \Phi^{*}\left(\xi_{2}^{-1}\right) \cdot D-\Sigma \cdot D \cdot\left(\Phi^{*}\left(\xi_{2}^{-1}\right)-D\right) .
\end{aligned}
$$

Since $\Phi(\Sigma)$ is 2 dimensional and $\xi_{2}$ is ample, one has that

$$
\operatorname{Deg}\left(\left.\Phi\right|_{\Sigma}\right) \Phi(\Sigma) \cdot\left(\xi_{2}^{-1}\right)^{2}=\operatorname{Deg}\left(\left.\Phi\right|_{\Sigma}\right) \Phi(\Sigma) \cdot\left(\xi_{2}\right)^{2}>0 .
$$

In order to conclude that $S \cdot \xi_{1}^{2}>0$ we show that (a) $\Sigma \cdot \Phi^{*}\left(\xi_{2}^{-1}\right) \cdot D \leq 0$ and that $\left(\right.$ b) $\Sigma \cdot D \cdot\left(\Phi^{*}\left(\xi_{2}^{-1}\right)-D\right) \leq 0$.

(a) Observe that $\Sigma$ cuts out an effective divisor on $D$, namely $\Sigma \cdot D$, which we denote by $\Delta=: \sum m_{\alpha} \Delta_{\alpha}$. Hence

$$
\Sigma \cdot \Phi^{*}\left(\xi_{2}^{-1}\right) \cdot D=\Delta \cdot \Phi^{*}\left(\xi_{2}^{-1}\right)=: \sum_{\alpha} m_{\alpha}\left(\operatorname{deg}\left(\left.\Phi\right|_{\Delta_{\alpha}}\right)\right) \Phi\left(\Delta_{\alpha}\right) \cdot \xi_{2}^{-1} \leq 0
$$

since $\xi_{2}$ is ample and $\Phi\left(\Delta_{\alpha}\right)$ is either a curve or a point.

(b) With $\Delta=\Sigma \cdot D=: \sum m_{\alpha} \Delta_{\alpha}$ as in (a) we have that

$$
\begin{aligned}
\Sigma \cdot D \cdot\left(\Phi^{*}\left(\xi_{2}^{-1}\right)-D\right) & =\Delta \cdot\left(\Phi^{*}\left(\xi_{2}^{-1}\right)-D\right)=\Delta \cdot\left(b^{*}\left(\xi_{1}^{-1}\right)\right) \\
& =\sum m_{\alpha}\left(\operatorname{deg}\left(\left.b\right|_{\Delta_{\alpha}}\right)\right) b\left(\Delta_{\alpha}\right) \cdot \xi_{1}^{-1} .
\end{aligned}
$$

Now for each component $\Delta_{\alpha}$ of $\Delta$ there are three possibilities: $b\left(\Delta_{\alpha}\right)$ is a point, $b\left(\Delta_{\alpha}\right)=\widetilde{B}_{j}$ for some $j$ (where $\widetilde{B}_{j}$ denotes the curve in $\mathbf{P}\left(T^{*} X\right)$ corresponding to the curve $B_{j}$ and the normal bundle $\left.N_{B_{j}}\right)$, or finally $b\left(\Delta_{\alpha}\right) \approx \mathbf{P}^{1}$ is a fiber of $\mathbf{P}\left(T^{*} X\right)$. If $b\left(\Delta_{\alpha}\right)$ is a point, then $b\left(\Delta_{\alpha}\right) \cdot \xi_{1}^{-1}=0$. If $b\left(\Delta_{\alpha}\right)=\widetilde{B}_{j}$ for some $j$, then $b\left(\Delta_{\alpha}\right) \cdot \xi_{1}^{-1}=$ $\widetilde{B}_{j} \cdot \xi_{1}^{-1}=B_{j} \cdot N_{B_{j}}=B_{j} \cdot B_{j}<0$, by hypothesis. If $b\left(\Delta_{\alpha}\right) \approx \mathbf{P}^{1}$ is a fiber of $\mathbf{P}\left(T^{*} X\right)$, then $b\left(\Delta_{\alpha}\right) \cdot \xi_{1}^{-1}=\mathbf{P}^{1} \cdot O(-1)=-1$. We conclude that for all $\alpha b\left(\Delta_{\alpha}\right) \cdot \xi_{1}^{-1} \leq 0$ and hence from (2.5) that $\Sigma \cdot D \cdot\left(\Phi^{*}\left(\xi_{2}^{-1}\right)-D\right) \leq 0$.

Now (2.4) along with (a) and (b) gives that $S \cdot \xi_{1}^{2}>0$.

Case (2ii) $\Phi(\Sigma)$ is not 2 dimensional. This implies that $S$ is contained in $\rho^{-1}\left(B_{j}\right)$ for some $B_{j}$ in the ramification set $\bigcup B_{k}$. Then $S=\rho^{-1}\left(B_{j}\right)=\mathbf{P}\left(\left.T^{*} X\right|_{B_{j}}\right)$. Therefore:

$$
\begin{aligned}
\xi_{1}^{2} \cdot S & =\operatorname{deg}\left(\left.T^{*} X\right|_{B_{j}}\right):=\operatorname{deg}\left(\operatorname{det}\left(\left.T^{*} X\right|_{B_{j}}\right)\right) \quad \text { as in [3] } \\
& =\operatorname{deg}\left(\left.K_{X}\right|_{B_{j}}\right) \\
& =\left(K_{B_{j}}-B_{j}\right) \cdot B_{j} \quad \text { by adjunction } \\
& =K_{B_{j}} \cdot B_{j}-B_{j} \cdot B_{j}=-e\left(B_{j}\right)-B_{j} \cdot B_{j}>0 .
\end{aligned}
$$


The last inequality follows from the hypothesis that $B_{j} \cdot B_{j}<0$ along with a first remark of the proof (that $T^{*} Y$ ample implies $e\left(B_{j}\right)<0$ ).

Case (3) n=3. $V_{n}=\mathbf{P}\left(T^{*} X\right)$. We have that

$$
\begin{aligned}
&(2.6)-\mathbf{P}\left(T^{*} X\right) \cdot \xi_{1}^{3}=\mathbf{P}\left(T^{*} X\right) \cdot\left(\xi_{1}^{-1}\right)^{3}=\Pi\left(T^{*} X\right) \cdot\left(b^{*}\left(\xi_{1}^{-1}\right)\right)^{3} \\
&=\Pi\left(T^{*} X\right) \cdot\left(b^{*}\left(\xi_{1}^{-1}\right)\right)^{2} \cdot\left(\Phi^{*}\left(\xi_{2}^{-1}\right)-D\right) \\
&= \\
& \Pi\left(T^{*} X\right) \cdot\left(b^{*}\left(\xi_{1}^{-1}\right)\right)^{2} \cdot \Phi^{*}\left(\xi_{2}^{-1}\right) \\
&=-\Pi\left(T^{*} X\right) \cdot\left(b^{*}\left(\xi_{1}^{-1}\right)\right)^{2} \cdot D \\
&= \Pi\left(T^{*} X\right) \cdot\left(b^{*}\left(\xi_{1}^{-1}\right)\right)^{2} \cdot \Phi^{*} \cdot \Phi^{*}\left(\boldsymbol{\Phi}_{2}^{-1}\right)-\left(\xi_{2}^{*-1}\left(\xi_{1}^{-1}\right)\right)^{2} \cdot D \\
&-\left(\xi_{1}^{-1}\right)^{2} \cdot \sum_{\alpha} n_{\alpha} \operatorname{deg}\left(\left.b\right|_{D_{\alpha}}\right) b\left(D_{\alpha}\right) \\
&= \Pi\left(T^{*} X\right) \cdot\left(b^{*}\left(\xi_{1}^{-1}\right)\right)^{2} \cdot \Phi^{*}\left(\xi_{2}^{-1}\right) .
\end{aligned}
$$

The last equality follows since $b\left(D_{\alpha}\right)$ is one dimensional by Proposition 2.1. Furthermore:

$$
\begin{aligned}
& \Pi\left(T^{*} X\right) \cdot\left(b^{*}\left(\xi_{1}^{-1}\right)\right)^{2} \cdot \Phi^{*}\left(\xi_{2}^{-1}\right) \\
&=\Pi\left(T^{*} X\right) \cdot\left(\Phi^{*}\left(\xi_{2}^{-1}\right)-D\right) \cdot\left(b^{*}\left(\xi_{1}^{-1}\right)\right) \cdot \Phi^{*}\left(\xi_{2}^{-1}\right) \\
&= \Pi\left(T^{*} X\right) \cdot\left(\Phi^{*}\left(\xi_{2}^{-1}\right)\right) \cdot\left(b^{*}\left(\xi_{1}^{-1}\right)\right) \cdot \Phi^{*}\left(\xi_{2}^{-1}\right) \\
&-\Pi\left(T^{*} X\right) \cdot D \cdot\left(b^{*}\left(\xi_{1}^{-1}\right)\right) \cdot \Phi^{*}\left(\xi_{2}^{-1}\right) \\
&= \Pi\left(T^{*} X\right) \cdot\left(\Phi^{*}\left(\xi_{2}^{-1}\right)\right) \cdot\left(\Phi^{*}\left(\xi_{2}^{-1}\right)-D\right) \cdot \Phi^{*}\left(\xi_{2}^{-1}\right) \\
&-D \cdot\left(b^{*}\left(\xi_{1}^{-1}\right)\right) \cdot \Phi^{*}\left(\xi_{2}^{-1}\right) \\
&= \Pi\left(T^{*} X\right) \cdot\left(\Phi^{*}\left(\xi_{2}^{-1}\right)\right)^{3}-D \cdot\left(\Phi^{*}\left(\xi_{2}^{-1}\right)\right)^{2} \\
&-D \cdot\left(b^{*}\left(\xi_{1}^{-1}\right)\right) \cdot \Phi^{*}\left(\xi_{2}^{-1}\right) .
\end{aligned}
$$

We next show that the above term is negative by analyzing each summand. First

$$
\begin{aligned}
\Pi\left(T^{*} X\right) \cdot\left(\Phi^{*}\left(\xi_{2}^{-1}\right)\right)^{3} & =(\operatorname{deg} \Phi) \Phi\left(\Pi\left(T^{*} X\right)\right) \cdot\left(\xi_{2}^{-1}\right)^{3} \\
& =(\operatorname{deg} \Phi) \mathbf{P}\left(T^{*} Y\right) \cdot\left(\xi_{2}^{-1}\right)^{3}<0
\end{aligned}
$$

since $\xi_{2}$ is ample. Next, for each irreducible component $D_{\alpha}$ of $D$ one has that

$$
\begin{aligned}
D_{\alpha} \cdot\left(\Phi^{*}\left(\xi_{2}^{-1}\right)\right)^{2} & =\left(\left.\operatorname{deg} \Phi\right|_{D_{\alpha}}\right) \Phi\left(D_{\alpha}\right) \cdot\left(\xi_{2}^{-1}\right)^{2} \\
& =\left(\left.\operatorname{deg} \Phi\right|_{D_{\alpha}}\right) \Phi\left(D_{\alpha}\right) \cdot\left(\xi_{2}\right)^{2} \geq 0
\end{aligned}
$$


with positivity depending on the dimension of $\Phi\left(D_{\alpha}\right)$, since $\xi_{2}$ is ample. Therefore

$$
-D \cdot\left(\Phi^{*}\left(\xi_{2}^{-1}\right)\right)^{2}=-\sum n_{\alpha} D_{\alpha} \cdot\left(\Phi^{*}\left(\xi_{2}^{-1}\right)\right)^{2} \leq 0
$$

since $n_{\alpha} \geq 0$. Finally, by Proposition 2.3 below, we have that $D$. $\left(b^{*}\left(\xi_{1}^{-1}\right)\right) \cdot \Phi^{*}\left(\xi_{2}^{-1}\right) \geq 0$ giving by $(2.7)$ that $\Pi\left(T^{*} X\right) \cdot\left(b^{*}\left(\xi_{1}^{-1}\right)\right)^{2}$. $\Phi^{*}\left(\xi_{2}^{-1}\right)<0$. By $(2.6)$ one concludes that $\mathbf{P}\left(T^{*} X\right) \cdot \xi_{1}^{3}>0$. This finishes Case 3 and proves the converse, that $\xi_{1}$ is ample.

This completes the proof of Theorem 1.1.

Proposition 2.3. In the setting of Theorem 1.1,

$$
D \cdot\left(b^{*}\left(\xi_{1}^{-1}\right)\right) \cdot \Phi^{*}\left(\xi_{2}^{-1}\right) \geq 0 .
$$

Proof. Let $D=\sum n_{\alpha} D_{\alpha}$ with $n_{\alpha}>0 \quad \forall \alpha$. For each $D_{\alpha}$ there are three possibilities: $b\left(D_{\alpha}\right)$ in $\mathbf{P}\left(T^{*} X\right)$ is either a point, a fiber of $\rho$ (say $F_{\alpha} \approx \mathbf{P}^{1}$ ), or $\widetilde{B}_{j}$ for some $j$. If $b\left(D_{\alpha}\right)$ is a point then $D_{\alpha} \cdot\left(b^{*}\left(\xi_{1}^{-1}\right)\right) \cdot \Phi^{*}\left(\xi_{2}^{-1}\right)=0$ as $b^{*}\left(\xi_{1}^{-1}\right)$ is then trivial on $D_{\alpha}$.

If $b\left(D_{\alpha}\right)$ is a fiber $F_{\alpha}$ then on $D_{\alpha} b^{*}\left(\xi_{1}^{-1}\right) \approx b^{*}(O(-1)) \approx b^{*}\left(-p_{\alpha}\right)$ where $p_{\alpha}$ is a point on $F_{\alpha}$. Letting $G_{\alpha}$ be the (effective) divisor cut out by $b^{*}\left(p_{\alpha}\right)$ on $D_{\alpha}$, one has that

$$
D_{\alpha} \cdot\left(b^{*}\left(\xi_{1}^{-1}\right)\right) \cdot \Phi^{*}\left(\xi_{2}^{-1}\right)=-G_{\alpha} \cdot \Phi^{*}\left(\xi_{2}^{-1}\right)=-\Phi\left(G_{\alpha}\right) \cdot \xi_{2}^{-1} \geq 0
$$

since $\xi_{2}$ is ample.

Finally if $b\left(D_{\alpha}\right)=\widetilde{B}_{j}$ for some $j$, then on $D_{\alpha}$ one has that $b^{*}\left(\xi_{1}^{-1}\right) \approx b^{*} \rho^{*}\left(N_{B_{j}}\right)$. Furthermore, since $B_{j}$ is a Riemann surface with $B_{j} \cdot B_{j}<0$ then $c_{1}\left(N_{B_{j}}\right)=c_{1}\left(\sum_{k}-p_{j k}\right)$ where the $p_{j k}$ are points on $B_{j}$. Letting $D_{\alpha j k}$ be the divisor cut out on $D_{\alpha}$ by $b^{*} \rho^{*}\left(p_{j k}\right)$ we have that

$$
\begin{aligned}
D_{\alpha} \cdot\left(b^{*}\left(\xi_{1}^{-1}\right)\right) \cdot \Phi^{*}\left(\xi_{2}^{-1}\right)=D_{\alpha} \cdot b^{*} \rho^{*}\left(N_{B_{J}}\right) \cdot \Phi^{*}\left(\xi_{2}^{-1}\right) \\
\quad=D_{\alpha} \cdot b^{*} \rho^{*}\left(\sum_{k}-p_{j k}\right) \cdot \Phi^{*}\left(\xi_{2}^{-1}\right)=-\sum_{k} D_{\alpha j k} \cdot \Phi^{*}\left(\xi_{2}^{-1}\right) \\
\quad=-\sum_{k} \Phi\left(D_{\alpha j k}\right) \cdot \xi_{2}^{-1} \geq 0
\end{aligned}
$$

since $\xi_{2}$ is ample.

Hence $D \cdot\left(b^{*}\left(\xi_{1}^{-1}\right)\right) \cdot \Phi^{*}\left(\xi_{2}^{-1}\right)=\sum_{\alpha} n_{\alpha} D_{\alpha} \cdot\left(b^{*}\left(\xi_{1}^{-1}\right)\right) \cdot \Phi^{*}\left(\xi_{2}^{-1}\right) \geq 0$ as claimed. 
3. Examples. We provide in Example 3.1 a construction of such an $X$ as in Theorem 1.1. Then we expand Example 3.1 into a class of similar examples in Examples 3.2 and 3.3. These are all degree 2 branched covers of a surface $Y$ with ample $T^{*} Y$. Good background references for this section are [6], [8], and [2]. We recall that given any $n \geq 2$ and any arrangement $\Lambda$ of $k$ lines in complex projective 2 space $\mathbf{P}^{2}$, Hirzebruch [6] constructed the compact complex surface $H(\Lambda, n)$ which is the minimal desingularization of the singular surface associated to the function field:

$$
\mathbf{C}\left(z_{1} / z_{0}, z_{2} / z_{0}\right)\left(\sqrt[n]{l_{2} / l_{1}}, \sqrt[n]{l_{3} / l_{1}}, \ldots, \sqrt[n]{l_{k} / l_{1}}\right)
$$

where $\left(z_{0}, z_{1}, z_{2}\right)$ are homogeneous coordinates for $\mathbf{P}^{2}$ and $l_{j}=0$ is the equation of the $j$ th line in $\Lambda . H(\Lambda, n)$ is a branched covering of $\mathbf{B P}^{2}$, the blowup of $\mathbf{P}^{2}$ at each point $p$ with $r_{p} \geq 3$ where $r_{p}=$ $\#\{L \in \Lambda \mid p \in L\}$. The branch locus in $\mathbf{B P}^{2}$ consists of the set of proper transforms of lines in $\Lambda$ along with the exceptional curves in $\mathbf{B P}^{2}$. The branching order is $n$ above the branch locus. Sommese [8] characterized the $H(\Lambda, n)$ with ample cotangent bundle as satisfying:

(a) for any $L \in \Lambda, \#\left\{p \in L \mid r_{p} \geq 3\right\} \geq 2$ and

(b) if $n=3$ then $t_{3}=0$, while if $n=2$ then $t_{3}=t_{4}=0$, where $t_{r}=\#\left\{p \in \mathbf{P}^{2} \mid r_{p}=r\right\}$. In Examples 3.1-3.3 that follow, we will assume $n \geq 4$ and avoid concern with condition (b).

We will make use of the Chern numbers of $H(\Lambda, n)$, which have been computed in [6], [8]. If one defines $f_{0}:=\sum_{r=2}^{k} t_{r}$ and $f_{1}:=$ $\sum_{r=2}^{k} r t_{r}$ then one has:

$$
\begin{gathered}
c_{1}^{2}(H(\Lambda, n))=n^{k-3}\left[n^{2}\left(-5 k+9+3 f_{1}-4 f_{0}\right)\right. \\
\left.+4 n\left(k+f_{0}-f_{1}\right)+f_{1}-f_{0}+k+t_{2}\right] \\
c_{2}(H(\Lambda, n))=n^{k-3}\left[n^{2}\left(3-2 k+f_{1}-f_{0}\right)\right. \\
\left.+2 n\left(k+f_{0}-f_{1}\right)+f_{1}-t_{2}\right] .
\end{gathered}
$$

EXAMPLe 3.1. Choose six points $p_{i}, i=1, \ldots, 6$, in $\mathbf{P}^{2}$ satisfying four collinearity conditions on the $p_{i}$ as follows:

(a) $p_{1}, p_{2}$, and $p_{3}$ are collinear along the line $L_{1}$.

(b) $p_{1}, p_{4}$, and $p_{5}$ are collinear along the line $L_{2}$.

(c) $p_{2}, p_{4}$, and $p_{6}$ are collinear along the line $L_{3}$.

(d) $p_{3}, p_{5}$, and $p_{6}$ are collinear along the line $L_{4}$.

Figure 1 represents this pattern succinctly. 


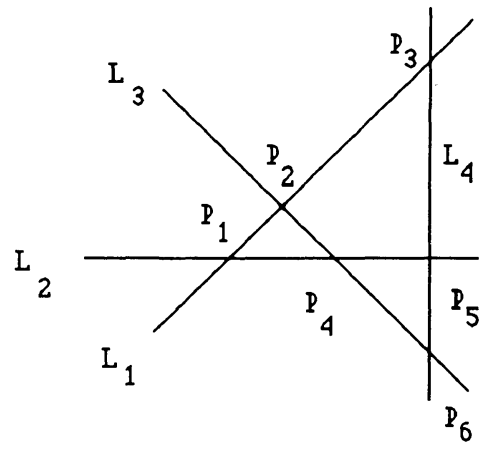

FIGURE 1

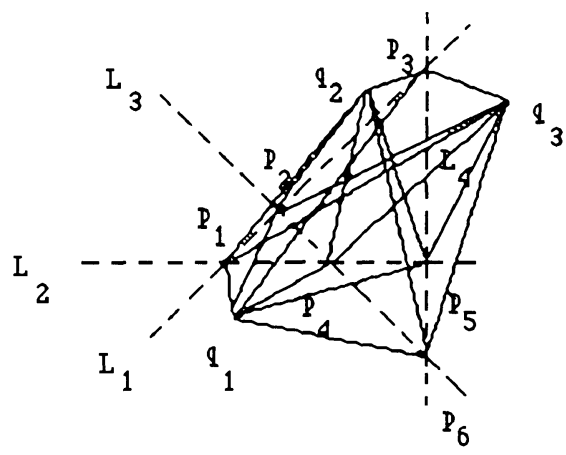

FIGURE 2

Next we choose an arrangement of lines $\Lambda$ in $\mathbf{P}^{2}$, satisfying:

(1) The above lines of collinearity are not in $\Lambda$ (i.e. $L_{j} \notin \Lambda$ for $j=1, \ldots, 4)$.

(2) $r_{p_{i}} \geq 3$ for each point $p_{i}, i=1, \ldots, 6$.

(3) For any other point $p \in L_{1} \cup L_{2} \cup L_{3} \cup L_{4} \backslash\left\{p_{1}, p_{2}, p_{3}, p_{4}, p_{5}, p_{6}\right\}$ one has $r_{p} \leq 1$.

(4) For each $L \in \Lambda$ at least 2 points $p$ of $L$ satisfy $r_{p} \geq 3$. An explicit example of such an arrangement $\Lambda$ is given in Figure 2 . The $\Lambda$ in Figure 2 is obtained by choosing three generic points, say $q_{1}, q_{2}, q_{3}$, not on $\bigcup L_{j}$. To each $q_{i}$ form the "pencil" of the six lines through $q_{i}$ containing the $p_{j}, j=1, \ldots, 6$. The resulting arrangement of 18 lines $\Lambda$ will be called a threefold cone on $p_{1}, p_{2}, p_{3}, p_{4}$, $p_{5}, p_{6}$ and it will satisfy all the above assumptions (1) through (4), provided (as in the generic choice of $q_{1}, q_{2}, q_{3}$ ) that there are no points $p$ on both the threefold cone and $\bigcup L_{j}$ with $r_{p} \geq 2$, other than $p_{1}, p_{2}, p_{3}, p_{4}, p_{5}, p_{6}$. (One can similarly construct $a$-fold cones on the $q=6$ points, where $a \geq 3$ : pick $a$ points not among the original $q$ and run lines from each of the $a$ points to each of the $q$ points. This is a line arrangement consisting of $a$ "pencils" with $q$ lines in each "pencil".)

Given such an arrangement $\Lambda$ satisfying (1)-(4), blowup all points with $r_{p} \geq 3$ to get $\mathbf{B P}^{2}$, which is branch covered by $H(\Lambda, n)$. For $n \geq 4, H(\Lambda, n)$ has ample cotangent bundle [8]. We pick $Y=H(\Lambda, n)$ as the base of our branched cover $X$, which we next construct. 
Let $H$ denote the pullback of the hyperplane in $\mathbf{P}^{2}$ to $\mathbf{B P}^{2}$. Let $L^{\prime}$ denote the proper transform of $L$ in $\mathbf{B P}^{2}$. Let $E_{i}$ denote the exceptional curve in $\mathbf{B P}^{2}$ over $p_{i}$ in $\mathbf{P}^{2}$ for $i=1, \ldots, 6$. Then we have that on $\mathbf{B P}^{2}$ :

(1) $L_{1}^{\prime}$ is linearly equivalent to $H-E_{1}-E_{2}-E_{3}$,

(2) $L_{2}^{\prime}$ is linearly equivalent to $H-E_{1}-E_{4}-E_{5}$,

(3) $L_{3}^{\prime}$ is linearly equivalent to $H-E_{2}-E_{4}-E_{6}$,

(4) $L_{4}^{\prime}$ is linearly equivalent to $H-E_{3}-E_{5}-E_{6}$.

Hence $\sum_{i=1}^{4} L_{i}^{\prime}$ is linearly equivalent to $4 H-2 \sum_{i=1}^{6} E_{i}=$ $2\left(2 H-\sum_{i=1}^{6} E_{i}\right)$. Therefore the line bundle corresponding to $\sum_{i=1}^{4} L_{i}^{\prime}$ has a square root, and this fact provides a smooth branched cover $Z$ of $\mathbf{B P}^{2}$ of degree 2, which is branched precisely over $\bigcup_{i=1}^{4} L_{i}^{\prime}$ (see $\mathrm{p}$. 42 of [1]).

Let $c: H(\Lambda, n) \rightarrow \mathbf{B P}^{2}$ denote the branched covering due to Hirzebruch, and $C: Z \rightarrow \mathbf{B P}^{2}$ denote the above constructed branched covering. Take $X$ to be $C^{*}(H(\Lambda, n))=H(\Lambda, n) \times_{\mathbf{B P}^{2}} Z=\{(r, q) \in$ $H(\Lambda, n) \times Z \mid c(r)=C(q)\}$. Then for $i=1, \ldots, 4$, transversality of $L_{i}^{\prime}$ to the branch locus of $c$ in $\mathbf{B P}^{2}$ (i.e. to $\Lambda \cup$ exceptional curves) gives that $X$ is smooth. Furthermore first factor projection $\pi_{1}: X \rightarrow$ $H(\Lambda, n)$ exhibits $X$ as a branched covering of $H(\Lambda, n)$ with ramification set $\bigcup B_{j}$ where the union ranges over $B_{j}$ in $\left(c \circ \pi_{1}\right)^{-1}\left(\bigcup_{i=1}^{4} L_{i}^{\prime}\right)$. This implies that $B_{j} \cdot B_{j}<0$ since $L_{i}^{\prime} \cdot L_{i}^{\prime}=-2$. By Theorem 1.1, $T^{*} X$ is ample. Note that $c \circ \pi_{1}: X \rightarrow \mathbf{B P}^{2}$ cannot be an $f: H\left(\Lambda^{*}, n^{*}\right) \rightarrow$ $\mathbf{B P}^{2}$ for any $\Lambda^{*}$ or any $n^{*}$, since the branching order in $H\left(\Lambda^{*}, n^{*}\right)$ is always $n^{*}$, while we have branching orders of 2 along the $B_{j}$ and $n \geq 4$ along $\left(c \circ \pi_{1}\right)^{-1}\left(\Lambda^{\prime} \cup\right.$ exceptional curves $)$.

From formulas (3.4) in Example 3.2 we have the Chern numbers $c_{2}(X)$ and $c_{1}^{2}(X)$ for the double cover $X$ of $H(\Lambda, n)$ where $\Lambda$ is the $a$-fold cone on the $q=6$ points $p_{1}, \ldots, p_{6}$. For $a \neq 6$ these are given by:

$$
\begin{aligned}
c_{2}(X)= & 2 n^{6 a-1}\left(15 a^{2}-10 a-1\right) \\
& -4 n^{6 a-2}\left(15 a^{2}-7 a-3\right)+6 n^{6 a-3}\left(5 a^{2}-a\right), \\
c_{1}^{2}(X)= & 2 n^{6 a-1}\left(30 a^{2}-16 a-5\right) \\
& -8 n^{6 a-2}\left(15 a^{2}-7 a-3\right)+2 n^{6 a-3}\left(30 a^{2}-13 a-6\right) .
\end{aligned}
$$

In particular for the 3 -fold cone on the $q=6$ points we have: 


$$
\begin{aligned}
& c_{2}(X)=n^{15}\left(208 n^{2}-444 n+252\right), \\
& c_{1}^{2}(X)=n^{15}\left(434 n^{2}-888 n+450\right) .
\end{aligned}
$$

When $n=5$ this reduces to $c_{2}(X)=5^{15}(3232)=5^{15} 2^{5} 101$ and $c_{1}^{2}(x)=5^{15}(6860)=5^{16} 2^{2} 7^{3}$. We show that when $n=5, a=3$, and $q=6$ (for our 6 points $p_{1}, \ldots, p_{6}$ ) that the double cover $X$ cannot be any one of Hirzebruch's surfaces $H\left(\Lambda^{\prime}, m\right)$ (for any $m$ and any line arrangement $\left.\Lambda^{\prime}\right)$. If $X$ were an $H\left(\Lambda^{\prime}, m\right)$ then by (3.1)

$$
\begin{aligned}
& c_{1}^{2}(X)=m^{k-3}[ m^{2}\left(-5 k+9+3 f_{1}-4 f_{0}\right) \\
&\left.+4 m\left(k+f_{0}-f_{1}\right)+f_{1}-f_{0}+k+t_{2}\right] \\
& c_{2}(X)=m^{k-3}\left[m^{2}\left(3-2 k+f_{1}-f_{0}\right)+2 m\left(k+f_{0}-f_{1}\right)+f_{1}-t_{2}\right]
\end{aligned}
$$

giving that $m^{k-3}$ divides both $c_{2}(X)=5^{15} 2^{5} 101$ and $c_{1}^{2}(X)=$ $5^{16} 2^{2} 7^{3}$. This implies that either $k=3$ and $m$ is arbitrary, or $k>3$ and $m=5^{b} 2^{c}$ for some $b, c$ (where the restrictions $0 \leq b(k-3) \leq 15$ and $0 \leq c(k-3) \leq 2$ must hold). The case $k=3$ and $m$ arbitrary is easily ruled out as $k=3$ lines do not produce the appropriate Chern numbers. In the case that $k>3$ we have that $k, b$, and $c$ are bounded, and a computer search on the possible cases for $k, b$, and $c$ on formal line arrangements rules out any case with

$$
\frac{c_{1}^{2}}{c_{2}}=\frac{5^{16} 2^{2} 7^{3}}{5^{15} 2^{5} 101}=2.122 \overline{5247}
$$

A formal line arrangement is taken here to mean a tuple of nonnegative integers $\left(t_{2}, t_{3}, \ldots, t_{k}\right)$ where

$$
\left(\begin{array}{l}
k \\
2
\end{array}\right)=\sum_{r=2}^{k} t_{r}\left(\begin{array}{l}
r \\
2
\end{array}\right)
$$

must hold. Thus we see that in the case that $n=5, a=3$, and $q=6$, we have a surface with ample cotangent bundle which cannot be one of Hirzebruch's surfaces $H\left(\Lambda^{\prime}, m\right)$.

By computing values for (3.2) and letting $n \geq 4$ and $a \geq 3$ one sees that the various Chern ratios for the double covers (of the $H(\Lambda, n)$ for $\Lambda$ the $a$-fold cone over 6 points) range from a high of $c_{1}^{2} / c_{2} \approx 2.12971$ (when $n=4$ and $a=3$ ) down through $c_{1}^{2} / c_{2} \approx 2$ asymptotically. 
EXAMPLE 3.2. We can generalize the above construction in the following way. Replace the lines $L_{i}, i=1, \ldots, 4$ (which we interpet as two "pencils" of two lines each) by $p \geq 2$ "pencils" $P_{i}$ (for $i=1, \ldots, p)$ in general position, with each "pencil" $P_{i}$ consisting of an even number, say $2 k_{i}$, of lines through a fixed point $p_{i}$. Choose a line arrangement $\Lambda$ as before satisfying the analogues of (1)-(4) in Example 3.1: each line in a "pencil" is not a line in $\Lambda$, if any two lines of the "pencils" intersect at a point $p$ then $r_{p} \geq 3$ in $\Lambda$, away from the intersection points of the lines in the "pencils" at most one line of $\Lambda$ passes through a point of the "pencils" (and it does so transversely), each line in $\Lambda$ contains at least 2 points $p$ with $r_{p} \geq 3$. Again, a generic $a$-fold cone on the $q$ points of intersection of the lines in the "pencils" will provide an explicit example of such a $\Lambda$.

Given such a $\Lambda$, let $Y=H(\Lambda, n)$ for $n \geq 4$ and let $Z$ be the double cover of $\mathbf{B P}^{2}$ corresponding to $\sum L_{j}^{\prime}=\left(\sum_{i} 2 k_{i}\right) H-\left(\sum n_{\alpha} E_{\alpha}\right)$. Here the $L_{j}^{\prime}$ are the proper transforms of all the lines in the $p$ "pencils", $H$ is the pullback of the hyperplane to $\mathbf{B P}^{2}$, and the $E_{\alpha}$ are the exceptional curves over the intersections of the lines in the "pencils" and the $n_{\alpha}$ denote the multiplicity of the $\alpha$ th intersection. Since each $n_{\alpha}$ is even (in fact $n_{\alpha}=2$ or $2 k_{i}$ ) $\sum L_{j}^{\prime}$ has a square root, yielding $Z$ a degree two branched cover of $\mathbf{B P}^{2}$ as in Example 3.1. For $n \geq 4$, take $X=H(\Lambda, n) \times_{\mathbf{B P}^{2}} Z$, which branch covers $H(\Lambda, n)$, with the branching of $\pi_{1}: X \rightarrow H(\Lambda, n)$ occurring on $\left(c \circ \pi_{1}\right)^{-1}\left(\bigcup L_{j}^{\prime}\right)$. Since $L_{j}^{\prime} \cdot L_{j}^{\prime}<0, T^{*} X$ is ample by Theorem 1.1 .

We begin computation of the Chern numbers of the surface $X$ which double covers $H(\Lambda, n)$ where $\Lambda$ is a generic $a$-fold cone on the

$$
q=p+\left(\begin{array}{c}
\sum_{i=1}^{p} 2 k_{i} \\
2
\end{array}\right)-\sum_{i=1}^{p}\left(\begin{array}{c}
2 k_{i} \\
2
\end{array}\right)
$$

intersection points of the $p$ "pencils". To be more explicit we rename $H(\Lambda, n)$ to be $H(\Lambda, a, q, n)$, the Hirzebruch line-arrangement surface constructed with the line-arrangement $\Lambda$ consisting of an $a$-fold cone on $q$ points, $a \neq q$, with branching order $n$. There are $k=a q$ lines in $\Lambda$. Let $L_{i j}$ be the $j$ th line in the $i$ th pencil and let $B_{i j}$ be the preimage in $H(\Lambda, a, q, n)$ of the proper transform $L_{i j}^{\prime}$ of $L_{i j}$. Then using the Hurwitz formula one has the euler number $e\left(B_{i j}\right)$ in 
$H(\Lambda, a, q, n)$ given by

$$
e\left(B_{i j}\right)=n^{a q-1}(2)-\left(n^{a q-1}-n^{a q-2}\right)\left[a q+\left(1+\sum_{l \neq i} 2 k_{l}\right)(1-a)\right]
$$

for each $j=1, \ldots, 2 k_{i}$. Since $\left(L_{i j}^{\prime}\right)^{2}=-2\left(\sum_{l \neq i} k_{l}\right)$ we have that $B_{i j}^{2}=-2\left(\sum_{l \neq i} k_{l}\right) n^{a q-1}$ for $j=1, \ldots, 2 k_{i}$.

For any line-arrangement $\Lambda$ which is an $a$-fold cone on $q$ points (where $a \neq q$ ), we have that $k=a q, t_{q}=a, t_{a}=q$, and $t_{2}=\left(\begin{array}{l}k \\ 2\end{array}\right)-$ $\sum_{r=3}^{k} t_{r}\left(\begin{array}{l}r \\ 2\end{array}\right)$, from which we have that $f_{0}=t_{2}+t_{a}+t_{q}=\left(\begin{array}{c}a q \\ 2\end{array}\right)-q\left(\begin{array}{c}a \\ 2\end{array}\right)-$ $a\left(\begin{array}{c}q \\ 2\end{array}\right)+q+a$ while $f_{1}=2 t_{2}+a t_{a}+q t_{q}=2\left(\left(\begin{array}{c}a q \\ 2\end{array}\right)-q\left(\begin{array}{c}a \\ 2\end{array}\right)-a\left(\begin{array}{c}q \\ 2\end{array}\right)\right)+a q+q a$. Using (3.1) one computes that:

(3.3) $c_{2}(H(\Lambda, a, q, n))$

$$
\begin{aligned}
=n^{a q-3}\left\{n ^ { 2 } ( 1 / 2 ) \left[a^{2} q(q-1)\right.\right. & \left.-a\left(q^{2}-q+2\right)-2 q+6\right] \\
-n\left[a^{2} q(q-1)-\right. & \left.a\left(q^{2}-3 q+2\right)-2 q\right] \\
& +(1 / 2)[a q(a(q-1)-q+5)]\}
\end{aligned}
$$

$$
\begin{aligned}
& c_{1}^{2}(H(\Lambda, a, q, n)) \\
& =n^{a q-3}\left\{n^{2}\left[a^{2} q(q-1)-a\left(q^{2}-2 q+4\right)-4 q+9\right]\right. \\
& -2 n\left[a^{2} q(q-1)-a\left(q^{2}-3 q+2\right)-2 q\right] \\
& \left.+\left[a^{2} q(q-1)-a\left(q^{2}-4 q+1\right)-q\right]\right\} .
\end{aligned}
$$

These allow computation of the Chern numbers of the double cover $X$ of $H(\Lambda, a, q, n)$ via the fact that $c_{2}(X)=2 c_{2}(H(\Lambda, a, q, n))-$ $(2-1) \sum_{i=1}^{p} \sum_{j=1}^{2 k_{i}} e\left(B_{i j}\right)$ and (letting $\pi_{1}: X \rightarrow H(\Lambda, a, q, n)$ be our branched covering and $K$ be the canonical bundle of $H(\Lambda, a, q, n)$ )

$$
\begin{aligned}
c_{1}^{2}(X) & =\pi_{1}^{*}\left(K+\sum_{i=1}^{p} \sum_{j=1}^{2 k_{i}}(1-(1 / 2)) B_{i j}\right)^{2} \\
& =2\left(K^{2}+\sum_{i=1}^{p} \sum_{j=1}^{2 k_{i}} K \cdot B_{i j}+(1 / 4) \sum_{i=1}^{p} \sum_{j=1}^{2 k_{i}} B_{i j}^{2}\right) .
\end{aligned}
$$


Using $K \cdot B_{i j}=-e\left(B_{i j}\right)-B_{i j}^{2}$ we obtain

$$
\begin{aligned}
& c_{2}(X)=2 c_{2}(H(\Lambda, a, q, n)) \\
& -\sum_{i=1}^{p} 2 k_{i}\left\{n^{a q-1}(2)-\left(n^{a q-1}-n^{a q-2}\right)\left[a q+\left(1+\sum_{l \neq i} 2 k_{l}\right)(1-a)\right]\right\} \\
& =n^{a q-1}\left[a^{2} q(q-1)-a\left(q^{2}-q+2\right)-2 q+6-4 \sum_{i=1}^{p} k_{i}\right. \\
& \left.+\sum_{i=1}^{p} 2 k_{i}\left[a q+\left(1+\sum_{l \neq i} 2 k_{l}\right)(1-a)\right]\right] \\
& +n^{a q-2}\left[-2 a^{2} q(q-1)+2 a\left(q^{2}-3 q+2\right)\right. \\
& \left.+4 q-\sum_{i=1}^{p} 2 k_{i}\left[a q+\left(1+\sum_{l \neq i} 2 k_{l}\right)(1-a)\right]\right] \\
& +n^{a q-3}[a q(a(q-1)-q+5)] \\
& c_{1}^{2}(X)=2\left\{c_{1}^{2}(H(\Lambda, a, q, n))\right. \\
& +n^{a q-1}\left[-4\left(\sum_{i=1}^{p} k_{i}\right)\right. \\
& \left.+2\left(\sum_{i=1}^{p} k_{i}\left[a q+\left(1+\sum_{l \neq i} 2 k_{l}\right)(1-a)\right]\right)+3 \sum_{i=1}^{p} \sum_{l \neq i} k_{i} k_{l}\right] \\
& \left.+n^{a q-2}\left[-2\left(\sum_{i=1}^{p} k_{i}\left[a q+\left(1+\sum_{l \neq i} 2 k_{l}\right)(1-a)\right]\right)\right]\right\} \\
& =n^{a q-1} 2\left[a^{2} q(q-1)-a\left(q^{2}-2 q+4\right)-4 q+9-4\left(\sum_{i=1}^{p} k_{i}\right)\right. \\
& \left.+2\left(\sum_{i=1}^{p} k_{i}\left[a q+\left(1+\sum_{l \neq i} 2 k_{l}\right)(1-a)\right]\right)+3 \sum_{i=1}^{p} \sum_{l \neq i} k_{i} k_{i}\right] \\
& +n^{a q-2} 2\left[-2 a^{2} q(q-1)+2 a\left(q^{2}-3 q+2\right)+4 q\right. \\
& \left.-2\left(\sum_{i=1}^{p} k_{i}\left[a q+\left(1+\sum_{l \neq i} 2 k_{l}\right)(1-a)\right]\right)\right] \\
& +n^{a q-3} 2\left[a^{2} q(q-1)-a\left(q^{2}-4 q+1\right)-q\right]
\end{aligned}
$$

where

$$
q=p+\left(\begin{array}{c}
\sum_{i=1}^{p} 2 k_{i} \\
2
\end{array}\right)-\sum_{i=1}^{p}\left(\begin{array}{c}
2 k_{i} \\
2
\end{array}\right) \text { and } a \neq q .
$$

In particular, if we let $p=2, k_{1}=k_{2}=1$, and $q=6$ then we obtain (3.2) in Example 3.1. 
EXAMPLE 3.3. One can make similar constructions by taking higher degree curves as well. Examples include:

(a) A "pencil" of $2 k$ lines $L_{1}, \ldots, L_{2 k}$ (through a common point $p_{1}$ ) and a smooth curve $C$ (in general position with respect to the "pencil"), along with a line arrangement $\Lambda$, where:

(i) each line $L_{i}$ in the "pencil" is not in $\Lambda$.

(ii) each point of intersection, $p$, of the $2 k$ lines or of the lines and curve, satisfies $r_{p} \geq 3$ in $\Lambda$.

(iii) away from $\left\{p_{1}\right\} \cup\left\{L_{1} \cap C\right\} \cup\left\{L_{2} \cap C\right\} \cup \cdots \cup\left\{L_{2 k} \cap C\right\}$ the lines in $\Lambda$ meet $L_{1} \cup \cdots \cup L_{2 k} \cup C$ transversely at points $p$ with $r_{p}=1$.

(iv) For each $L \in \Lambda$ at least two points $p$ of $L$ satisfy $r_{p} \geq 3$.

(v) $C$ is of even degree $2 d$ in $\left|H^{2 d}\right|$ in $\mathbf{P}^{2}$ where $d<k$. A generic $a$-fold cone on $\left\{p_{1}\right\} \cup\left\{L_{1} \cap C\right\} \cup\left\{L_{2} \cap C\right\} \cup \cdots \cup\left\{L_{2 k} \cap C\right\}$ gives an explicit example of such $\Lambda$ for $a \geq 3$. Then $C^{\prime}+\sum_{j=1}^{2 k} L_{j}^{\prime}=$ $2\left((k+d) H-k E_{1}-\sum_{j>1} E_{j}\right)$ where $E_{1}$ is the exceptional curve over $p_{1}$ and the $E_{j}$ in the second summation are the exceptional curves over $\left\{L_{1} \cap C\right\} \cup\left\{L_{2} \cap C\right\} \cup \cdots \cup\left\{L_{2 k} \cap C\right\}$. Hence $C^{\prime}+\sum_{j=1}^{2 k} L_{j}^{\prime}$ has a square root. Construct the corresponding branched cover $Z$ of $\mathbf{B P}^{2}$ of degree 2, along with $X=H(\Lambda, n) \times_{\mathbf{B P}^{2}} Z$ where $X$ branch covers $H(\Lambda, n)$ and $n \geq 4$. Then $d<k$ gives $\left(C^{\prime}\right)^{2}<0$, and $T^{*} X$ is ample by Theorem 1.1.

The Chern numbers of $X$, the double cover of Hirzebruch's surface $H(\Lambda, a, q, n)$ associated to the $a$-fold cone on the $q$ points of intersection of the "pencil" and curve, are given by:

$$
\begin{aligned}
c_{2}(X)= & 2(H(\Lambda, a, q, n)) \\
& +n^{a q-1}\left[4 d^{2}-6 d+8 k d-2 k\right]+n^{a q-2}[-2 k-8 k d] \\
= & n^{a q-1}\left[a^{2} q(q-1)-a\left(q^{2}-q+2\right)\right. \\
& \left.\quad-2 q+6+4 d^{2}-6 d+8 k d-2 k\right] \\
& +n^{a q-2}\left[-2 a^{2} q(q-1)+2 a\left(q^{2}-3 q+2\right)+4 q-2 k-8 k d\right] \\
& +n^{a q-3}[a q(a(q-1)-q+5)], \\
c_{1}^{2}(X)= & 2\left\{c_{1}^{2}(H(\Lambda, a, q, n))\right. \\
& \left.\quad+n^{a q-1}\left[d^{2}-6 d+14 k d-2 k\right]+n^{a q-2}[-2 k-8 k d]\right\} \\
= & n^{a q-1} 2\left[a^{2} q(q-1)-a\left(q^{2}-2 q+4\right)\right. \\
& \left.\quad-4 q+9+d^{2}-6 d+14 k d-2 k\right] \\
& +n^{a q-2} 2\left[-2 a^{2} q(q-1)+2 a\left(q^{2}-3 q+2\right)+4 q-2 k-8 k d\right] \\
& +n^{a q-3} 2\left[a^{2} q(q-1)-a\left(q^{2}-4 q+1\right)-q\right]
\end{aligned}
$$


where $q=1+4 k d, a \neq q$, and $d<k$.

(b) A "pencil" of $2 k$ lines $L_{1}, \ldots, L_{2 k}$ (through a common point) and a "pencil" of $2 m$ smooth curves $C_{1}, \ldots, C_{2 m}$ of degree $d$ in $\left|H^{d}\right|$ in $\mathbf{P}^{2}$ (having base locus $d^{2}$ points) in general position with respect to the "pencil", where each point of intersection, $p$, (of the $2 k$ lines, of the lines and curve, or of the curves) satisfies $r_{p} \geq 3$ in a line-arrangement $\Lambda$, and the analogues of (i) through (iv) in (a) are also satisfied. $\sum C_{i}^{\prime}+\sum L_{j}^{\prime}$ has a square root. Construct the corresponding branched cover $Z$ of $\mathbf{B P}^{2}$ of degree 2, along with $X=H(\Lambda, n) \times_{\mathbf{B P}^{2}} Z$ where $X$ branch covers $H(\Lambda, n)$ and $n \geq 4$. Then $T^{*} X$ is ample by Theorem 1.1.

The Chern numbers for $X$, the double cover of Hirzebruch's surface $H(\Lambda, a, q, n)$ associated to the $a$-fold cone on the $q$ points of intersection of the $2 k$ lines and $2 m$ curves, are:

$$
\begin{aligned}
c_{2}(X)= & 2(H(\Lambda, a, q, n))+n^{a q-1}\left[-2 k-6 m d+8 k m d+4 m d^{2}\right] \\
& +n^{a q-2}\left[-2 k-8 k m d-2 m d^{2}\right] \\
= & n^{a q-1}\left[a^{2} q(q-1)-a\left(q^{2}-q+2\right)\right. \\
& \left.-2 q+6-2 k-6 m d+8 k m d+4 m d^{2}\right] \\
& +n^{a q-2}\left[-2 a^{2} q(q-1)+2 a\left(q^{2}-3 q+2\right)\right. \\
& \left.+4 q-2 k-8 k m d-2 m d^{2}\right] \\
& +n^{a q-3}[a q(a(q-1)-q+5)] \\
c_{1}^{2}(X)= & 2\left\{c_{1}^{2}(H(\Lambda, a, q, n))+n^{a q-1}\left[-2 k-6 m d+14 k m d+4 m d^{2}\right]\right. \\
= & \quad n^{a q-1} 2\left[a^{2} q(q-1)-a\left(q^{2}-2 q+4\right)-4 q\right. \\
& \left.\quad+9-2 k-6 m d+14 k m d+4 m d^{2}\right] \\
& +n^{a q-2} 2\left[-2 a^{2} q(q-1)+2 a\left(q^{2}-3 q+2\right)\right. \\
& +n^{a q-3} 2\left[a^{2} q(q-1)-a\left(q^{2}-4 q+2 k-8 k m d-2 m d^{2}\right]\right\}
\end{aligned}
$$

where $q=1+d^{2}+4 k m d$ and $a \neq q$.

(c) Two or more "pencils" each consisting of an even number of curves will also yield similar examples.

EXAMPle 3.4. In [8], Sommese constructs branched coverings $X$ of $H(\Lambda, 5)$ where $\Lambda$ is the $A_{1}(6)$ arrangement of lines. He uses 
the coverings to show that the possible Chern ratios $c_{1}^{2} / c_{2}$ between 2 and 3 are assumed. However these coverings do not have ample cotangent bundle. This follows from Theorem 1.1 after one observes that: $H\left(A_{1}(6), 5\right)$ fibers over a Riemann surface, the branch locus of each of Sommese's coverings in $H\left(A_{1}(6), 5\right)$ consists of smooth fibers with self-intersection 0 , and the ramification set in each covering $X$ also consists of smooth curves with self-intersection 0 .

Acknowledgment. This work was supported by an OCSLA grant from East Carolina University while the author was a Scholar in Residence at Duke University.

\section{REFERENCES}

[1] W. Barth, C. Peters and A. Van de Ven, Compact Complex Surfaces, Ergebnisse der Mathematik und ihrer Grenzgebiete 3. Folge, Band 4, Springer-Verlag (1984).

[2] G. Barthel, F. Hirzebruch and T. Höfer, Geradenkonfigurationen und Algebraische Flächen: Eine Veroffentlichung des Max Planck Institut fur Mathematik, Bonn, Aspekte der Mathematik Ser Vol. D4, Vieweg \& Sohn (1987).

[3] A. Beauville, Complex Algebraic Surfaces, London Mathematical Society Lecture Notes, vol. 68, Cambridge University Press (1983).

[4] R. Hartshorne, Ample vector bundles, Publ. Math. IHES, 29 (1966), 319-350.

[5] H. Hironaka, Resolution of singularities of an algebraic variety over a field of characteristic zero, I, II, Ann. of Math., 79 (1964), 109-326.

[6] F. Hirzebruch, Arrangements of lines and algebraic surfaces, Arithmetic and Geometry Vol. 2, Progress in Math., vol. 36, Birkhäuser, Boston-Basel-Stuttgart, (1983), 113-140.

[7] Y. Nakai, A criterion of an ample sheaf on a projective scheme, Amer. J. Math., 85 (1963), 14-26.

[8] A. Sommese, On the density of ratios of Chern numbers of algebraic surfaces, Math. Ann., 268 (1984), 207-221.

[9] M. J. Spurr, Nef cotangent bundles over line arrangements, Manuscripta Math., 62 (1988), 369-374.

[10] K. Ueno, Classification theory of algebraic varieties and compact complex surfaces, Lecture Notes in Math., vol. 439, Springer-Verlag, Heidelberg (1975).

Received June 4, 1991.

East Carolina University

GREENVILLE, NC 27858

E-mail address: mammspur@ecuvm1.bitnet mammspur@ecuvm.cis.ecu.edu 


\section{PACIFIC JOURNAL OF MATHEMATICS}

Founded by

E. F. BeCKenbach (1906-1982) F. Wolf (1904-1989)

\section{EDITORS}

Sun-Yung A. Chang

(Managing Editor)

University of California

Los Angeles, CA 90024-1555

chang@math.ucla.edu

\section{F. Michael Christ}

University of California

Los Angeles, CA 90024-1555

christ@math.ucla.edu

\section{Herbert Clemens}

University of Utah

Salt Lake City, UT 84112

clemens@math.utah.edu

\author{
THOMAS ENRIGHT \\ University of California, San Diego \\ La Jolla, CA 92093 \\ tenright@ucsd.edu \\ Nicholas ERcolanI \\ University of Arizona \\ Tucson, AZ 85721 \\ ercolani@math.arizona.edu \\ R. FINN \\ Stanford University \\ Stanford, CA 94305 \\ finn@gauss.stanford.edu \\ VAUGHAN F. R. JONES \\ University of California \\ Berkeley, CA 94720 \\ vfr@math.berkeley.edu
}

Steven KerckhofF

Stanford University

Stanford, CA 94305

spk@gauss.stanford.edu

Martin SCHARLEMANN

University of California

Santa Barbara, CA 93106

mgscharl@math.ucsb.edu

Harold Stark

University of California, San Diego

La Jolla, CA 92093

V. S. VARADARAJAN

University of California

Los Angeles, CA 90024-1555

vsv@math.ucla.edu

\section{SUPPORTING INSTITUTIONS}

\author{
UNIVERSITY OF ARIZONA \\ UNIVERSITY OF BRITISH COLUMBIA \\ CALIFORNIA INSTITUTE OF TECHNOLOGY \\ UNIVERSITY OF CALIFORNIA \\ UNIVERSITY OF MONTANA \\ UNIVERSITY OF NEVADA, RENO \\ NEW MEXICO STATE UNIVERSITY \\ OREGON STATE UNIVERSITY
}

UNIVERSITY OF OREGON

UNIVERSITY OF SOUTHERN CALIFORNIA

STANFORD UNIVERSITY

UNIVERSITY OF HAWAII

UNIVERSITY OF UTAH

WASHINGTON STATE UNIVERSITY

UNIVERSITY OF WASHINGTON 


\section{PACIFIC JOURNAL OF MATHEMATICS}

Volume $164 \quad$ No. $1 \quad$ May 1994

Entropy versus orbit equivalence for minimal homeomorphisms

M. MiChaEL BoYle and DAVID E. HANDELMAN

The hyperspaces of infinite-dimensional compacta for covering and cohomological dimension are homeomorphic

TADEUSZ WLADYSLAW DOBROWOLSKI and LEONARD RUBIN

The index of transversally elliptic operators for locally free actions

JefFrey Stephen Fox and Peter Evarts Haskell

Unit indices of some imaginary composite quadratic fields

MiKiHITO HIRABAYASHI

Periodic points on nilmanifolds and solvmanifolds

EDWARD KEPPELMANN

Branched coverings of surfaces with ample cotangent bundle

MiCHAEL JEROME SPURR

Evolutionary existence proofs for the pendant drop and $n$-dimensional 147 catenary problems

ANDREW GUY STONE

Lattices of Lipschitz functions

NiKOLAI ISAAC WEAVER

Correction to: "Trace rings for verbally prime algebras" 